\title{
Use of Normalized Correlation Function to Discriminate Outcome of Persistent Patients Undergoing Electrical Cardioversion
}

\author{
Olivier Meste ${ }^{1}$, Stef Zeemering ${ }^{2}$ Joël Karel $^{4}$, Theo Lankveld ${ }^{2,3}$, Ulrich Schotten ${ }^{2}$, Harry Crijns ${ }^{3}$, \\ Ralf Peeters ${ }^{4}$, Pietro Bonizzi ${ }^{4}$ \\ ${ }^{1}$ Laboratoire I3S, UNSA-CNRS, Nice, France \\ ${ }^{2}$ Department of Physiology, Maastricht University Medical Center, Maastricht, The Netherlands \\ ${ }^{3}$ Department of Cardiology, Maastricht University Medical Center, Maastricht, The Netherlands \\ ${ }^{4}$ Department of Data Science and Knowledge Engineering, Maastricht University, Maastricht, \\ The Netherlands
}

\begin{abstract}
Atrial activity $(A A)$ during atrial fibrillation $(A F)$ is a process characterized by different short- and long-term recurrent behaviors. In this work we hypothesize that the features derived from these behaviors contain an information on the lead locations that discriminate the most between persistent AF patients recurring after electrical cardioversion from those who do not. Body surface potential maps (BSPMs, 184 electrodes) were recorded in 63 patients in persistent $A F$ prior to electrical cardioversion $(32$ recurrences after 4-6 weeks). A correlation function (CF) was computed for each electrode, and normalized in order to make it independent of its magnitude. Finally, the first min and max values of the normalized $C F$ from each electrode were used to discriminate patients outcome. The corresponding spatial maps showed that electrodes with the largest values of normalized $C F$ are located on the front of the torso, centered around V1. A Wilcoxon rank-sum test was used to compare maps of recurrent and non-recurrent AF patients and find electrodes with significantly different magnitude. A significant difference was observed on the upper and lower parts of the torso, with higher values for the non-recurrent AF patients (higher recurrence of the underlying AA propagation patterns). When looking at the energy computed on each electrode, this parameter was not able to distinguish among the two groups.
\end{abstract}

\section{Introduction}

Although under the scope of many studies and researches, the characterization of atrial fibrillation (AF) is still an open field, especially when the goal is the stratification for guiding AF therapy [1]. This guidance could be the targeting of the ablation sites by using information recorded non-invasively. In [3],[2], we intended to com- pute a recurrence AA signal based on a multidimensional autocorrelation function. Thus, novel insights in AA during $\mathrm{AF}$ have been proposed, in line with the underlying cardiac electrophysiology. However, these outcomes don't allow by nature an accurate spatial analysis, e.g., at the level of the recording electrode.

In this study, we decompose the multidimensional correlation to introduce the role of individual correlation functions, i.e., for each electrode. Features are extracted from these functions and the corresponding spatial maps are compared. Results shows how relevant is this approach and paves the road to a non-invasive spatial discrimination of non-recurrent AF subjects from recurrent ones. Although this discrimination is not the ultimate goal, because it is based on spatial parameters it shows that relevant information can be extracted from each electrodes taken separately.

\section{Methods}

\subsection{BSPM data and pre-processing}

BSPMs were recorded in 63 patients in persistent AF using a custom configuration of 184 leads with 120 anterior and 64 posterior leads (ActiveTwo BSM Panels Carbon Electrodes, Biosemi B.V., The Netherlands). All patients underwent electrical cardioversion. After a blanking period from 4 to 6 weeks after procedure, 32 patients showed AF recurrence (labeled R) and 31 remained under sinus rhythm (labeled NR). ECGs were sampled at $256 \mathrm{~Hz}$. A one-minute segment was selected for each subject, low-quality leads were excluded (low signal-tonoise ratio, poor electrode contact, motion artefacts), and Wilsons Central Terminal was subtracted in line with conventional ECG analysis. After band-pass filtering the signals between 1 and $100 \mathrm{~Hz}$ (3rd order Chebyshev), QRST 
cancellation was performed using an adaptive singular value decomposition method, inspired by the approach in [4], with multiple QRST window templates defined using hierarchical clustering. The extracted atrial signals were post-filtered with a zerophase notch filter at $50 \mathrm{~Hz}$ to suppress power line noise, and with a $3 \mathrm{~Hz}$ zero-phase highpass filter (3rd order Chebyshev) to remove low-frequency residuals not related to (persistent) AF.

\subsection{Normalized Correlation Function}

As published in [3], the nonstationary properties of noninvasive AA can be investigate by using a multivariable autocorrelation function of the spatial AA oscillatory patterns which provides a patient's specific recurrence signal. The spatial information is provided by the multiple observations of a single behavior by using electrodes mapping the torso. The autocorrelation function is based on the computation of elements $R_{i, j}$ defined by :

$$
R_{i, j}=\frac{\mathbf{x}(i)^{T} \mathbf{x}(i+j-1)}{\|\mathbf{x}(i)\|_{2}\|\mathbf{x}(i+j-1)\|_{2}}
$$

with vector $\mathbf{x}(n), n=1, \ldots, N$ represents the overall spatial AA from all electrodes $l, l=1, \ldots, L$, for a given time $n$. The corresponding multivariable autocorrelation function is, in turn, given by :

$$
r(p)=\frac{1}{M} \sum_{i=1}^{M} R_{i, p+1}
$$

Where $M \mathrm{M}$ is the window size of the analysis $(\mathrm{M} \mathrm{N})$. This function has been shown to efficiently exhibit short and long term behaviors characterizing AA. In fact, the inner product $\mathbf{x}(i)^{T} \mathbf{x}(i+j-1)$ is a linear combination of the contribution of each electrodes, that is $\sum_{l} x_{l}(i) x_{l}(i+$ $j-1)$. Then $r(p)$ can be rewritten as :

$$
r(p)=\frac{1}{M} \sum_{i=1}^{M} \frac{\sum_{l} x_{l}(i) x_{l}(i+p)}{\|\mathbf{x}(i)\|_{2}\|\mathbf{x}(i+p)\|_{2}}
$$

From that expression it can be concluded that the global autocorrelation function is not simply the averaging of individual monodimensional autocorrelation function :

$$
r_{l}(p)=\frac{1}{M} \sum_{i=1}^{M} x_{l}(i) x_{l}(i+p)
$$

but rather a non trivial weighted contribution of them. However, it appears that since the $r_{l}(p)$ 's participate to the spatially computed information it is probable that they individually produce exploitable features to distinguish $\mathrm{R}$ from NR population. Each $r_{l}(p)$ being related to one electrode contribution, it is expected that these features will inform on the corresponding discrimination spatially defined. In order to get rid of the amplitude variabilities observed from one lead to another, a normalized version of (4) is preferred such that $r_{l}(0)=1$ for all $l$.

In line with the discriminative features proposed in [3], the first min and max values of all the normalized $r_{l}(p)$ 's are computed for each patients of the groups $\mathrm{R}$ and NR.

In addition to this analysis, the energy of each electrode is also computed to check its power in discriminating between $\mathrm{R}$ and NR. This comparison is consistent with the correlation analysis because in both cases a second order metric is involved. Note that for the non normalized correlation the energy is equal to $r_{l}(0)$.

\section{Analyses and Results}

Both analyses have been performed on the two groups of subjects, according to the $\mathrm{R}$ and NR classification. For the autocorrelation based method the two relevant features for each electrode and subject are the first $a b s(\mathrm{Min})$ and Max values. In order to exemplify the approach, in fig. (1) and fig. (2) are given all the $r_{l}(p)$ 's for a NR (\#29) and $\mathrm{R}$ (\#45) subjects, respectively.

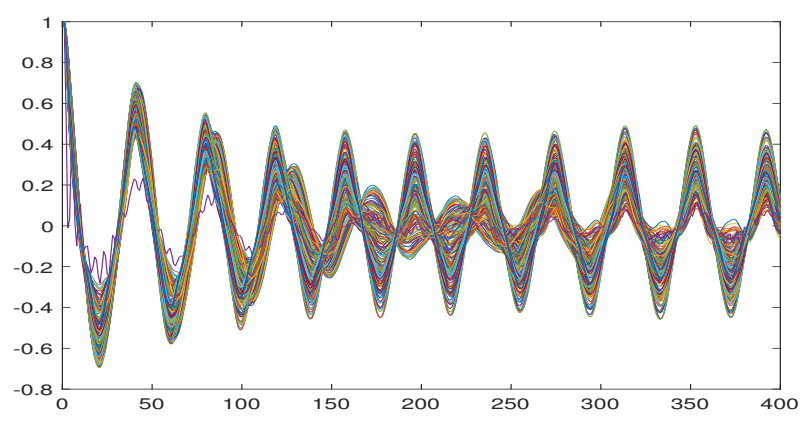

Figure 1. The $r_{l}(p)$ 's for the AF non-recurrent subject \#29

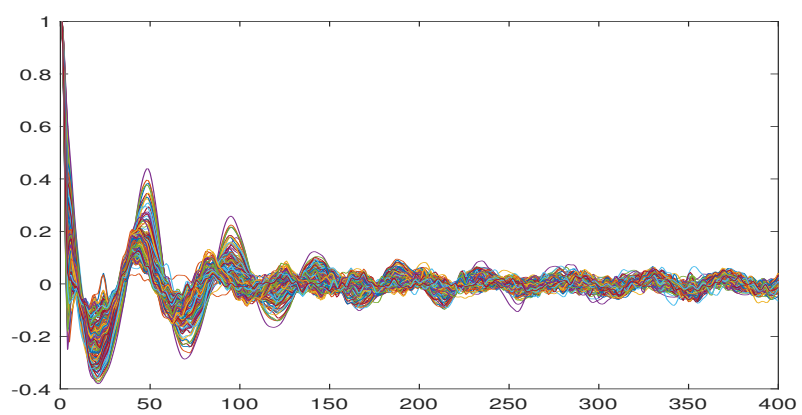

Figure 2. The $r_{l}(p)$ 's for the AF recurrent subject \#45

The corresponding spatial maps are given in fig. (3) and fig. (4) where a difference in magnitude and distribution is visible. The location of the maximum value of each map, marked by a bold black circle, is also different, but in the vicinity of $\mathrm{V} 1$. 


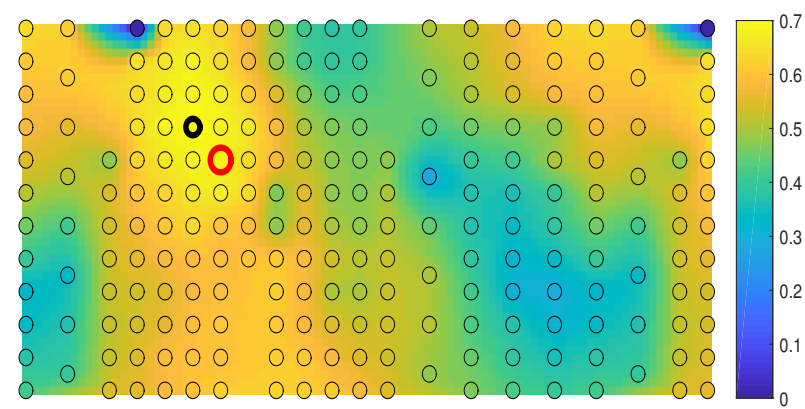

Figure 3. The spatial distribution of the $a b s(\mathrm{Min})$ values extracted from the $r_{l}(p)$ 's for the AF non-recurrent subject \#29. Red circle and bold black circle pinpoint the V1 electrode and the maximum value of the map, respectively.

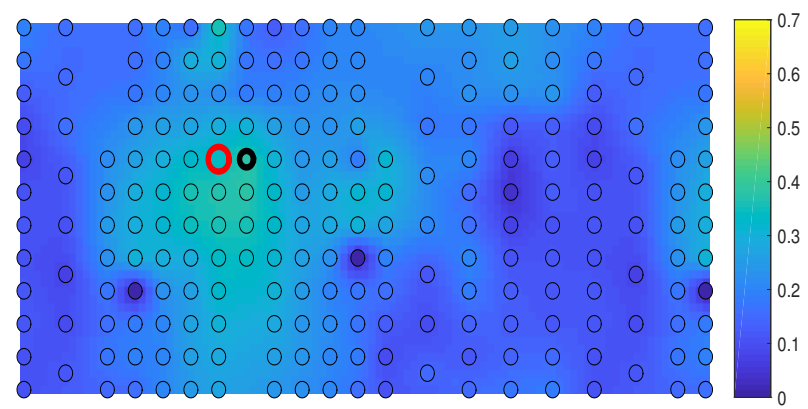

Figure 4. The spatial distribution of the abs(Min) values extracted from the $r_{l}(p)$ 's for a AF recurrent subject \#45. Red circle and bold black circle pinpoint the V1 electrode and the maximum value of the map, respectively.

In order to globally compare the two groups (R and NR), the median spatial maps for the two features (abs(Min) and $M a x$ ) and the two groups are given in fig. (5), fig. (6), fig. (7), fig. (8). Firstly, it can be noticed that regardless the mean map the greatest values are concentrated around V1, in agreement with the key role played by this electrode. Secondly, a Wilcoxon rank sum test for equal medians is performed to distinguish the electrodes with the highest power to discriminate the NR from the $\mathrm{R}$ groups. Even though the maps of the NR group tend to be higher than the ones from the R group (consistent with results in [3]), it appears that upper and lower electrodes significantly discriminate the most, covering the relevant area above the atria. It is worth noticing that the multiple comparisons are not corrected.

For the feature based on electrodes energy, spatial maps are given in fig. (9) and fig. (10), for the two groups. Similarly to the previous analysis, the energy is centered around electrode V1. There is a slight difference of the distributions but in that case the energy of the NR group doesn't tend to be higher, especially around V1 (not illustrated here). The Wilcoxon rank sum test for equal medians applied to all electrodes did not show any significant differences between the two groups.

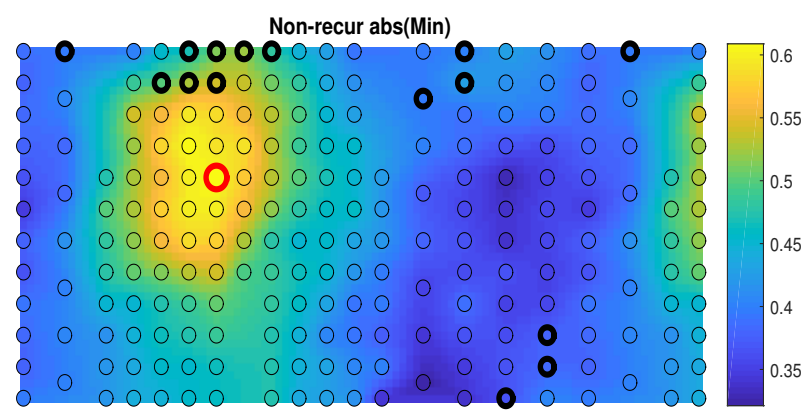

Figure 5. The mean spatial distribution of the $a b s(\mathrm{Min})$ values of the AF non-recurrent group. Red circle and bold black circles pinpoint the $\mathrm{V} 1$ electrode and the locations of significant $(\mathrm{p}<0.05)$ difference between NR and R groups, respectively.

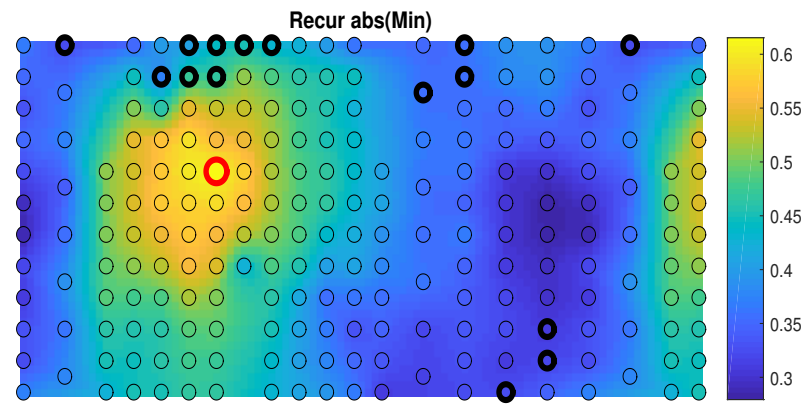

Figure 6. The mean spatial distribution of the $a b s(\mathrm{Min})$ values of the AF recurrent group. Red circle and bold black circles pinpoint the V1 electrode and the locations of significant $(\mathrm{p}<0.05)$ difference between NR and R groups, respectively.

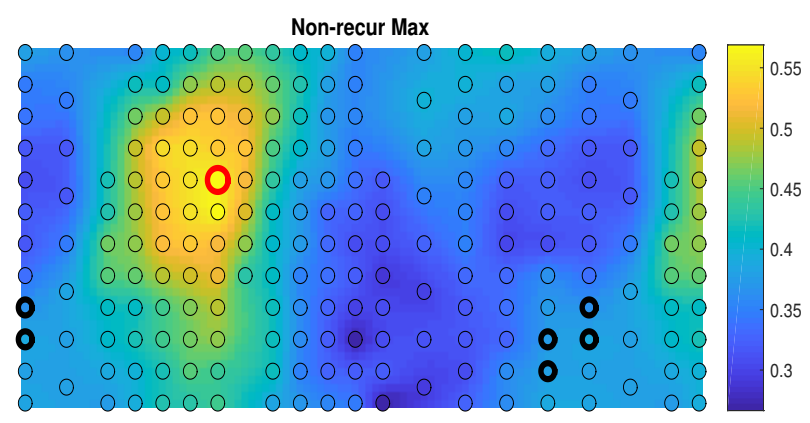

Figure 7. The mean spatial distribution of the Max values of the AF non-recurrent group. Red circle and bold black circles pinpoint the $\mathrm{V} 1$ electrode and the locations of significant $(\mathrm{p}<0.05)$ difference between NR and R groups, respectively.

\section{Conclusions}

This study proposed a spatial analysis of the autocorrelations computed from each electrode. The overall outcomes are in agreement with cardiac electrophysiology where information about AA during $\mathrm{AF}$ is expected to be better captured by V1. In addition, taken separately the proposed features emphasized the role of the upper and lower electrodes for the discrimination of $\mathrm{R}$ from NR subjects. For the upper electrodes, this could be explained by the prox- 


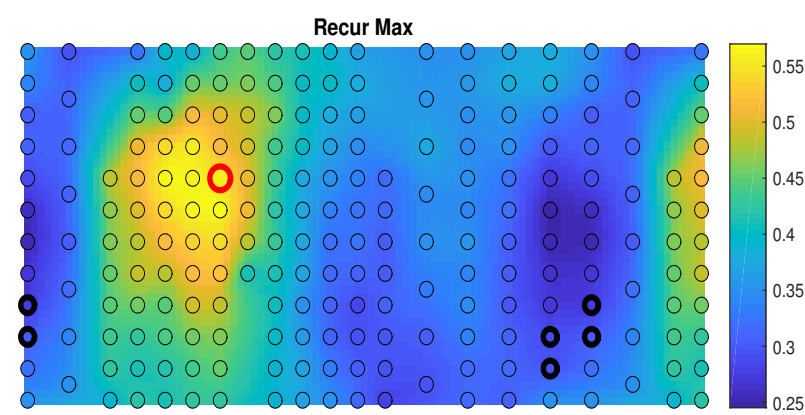

Figure 8. The mean spatial distribution of the Max values of the AF recurrent group. Red circle and bold black circles pinpoint the V1 electrode and the locations of significant $(p<0.05)$ difference between NR and R groups, respectively.

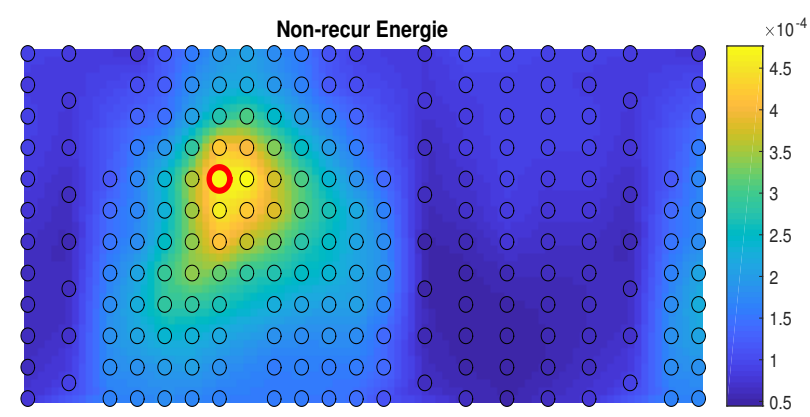

Figure 9. The mean spatial distribution of the Energy for the AF nonrecurrent group. Red circle pinpoints the V1 electrode.

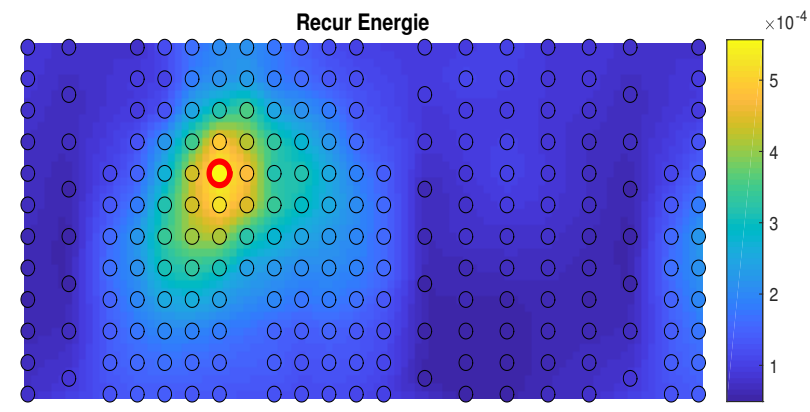

Figure 10. The mean spatial distribution of the Energy for the AF recurrent group. Red circle pinpoints the V1 electrode.

imity with the pulmonary veins often at the origin of AF. In contrast, the energy of each electrode failed for this discrimination. This could be explained by the inability of this metric to capture subtle variations unlike what is performed by the autocorrelation based features.

\section{References}

[1] Kirchhof P, Calkins H. Catheter ablation in patients with persistent atrial fibrillation. Eur Heart J ,2017;38(1):2026

[2] Bonizzi P, Zeemering S, Karel J, Azman M, Lankveld T, Schotten U, Crijns H, Peeters R, Meste O. Noninvasive characterisation of short- and long-term recurrence of atrial signals during persistent atrial fibrillation. Conf Proc Comp in Card, 2017;p 44

[3] Bonizzi P, Meste O, Zeemering S, Karel J, Lankveld T, et al.. A Novel Framework for Noninvasive Analysis of ShortTerm Atrial Activity Dynamics During Persistent Atrial Fibrillation. Medical and Biological Engineering and Computing, Springer Verlag, 2020;58:19331945.

[4] Alcaraz R, Rieta JJ. Adaptive singular value cancellation of ventricular activity in single-lead atrial fibrillation electrocardiograms. Physiol Meas, 2008;29:13511369.

Address for correspondence:

Pr. Olivier MESTE

Laboratoire I3S - CNRS - UNS

2000 route des Lucioles

06903 Sophia Antipolis cedex, FRANCE

E-mail address: olivier.meste@univ-cotedazur.fr 